\title{
Supplementary Material: Infinite Mixtures of Infinite Factor Analysers
}

\author{
Keefe Murphy, Cinzia Viroli and Isobel Claire Gormley
}

\section{Appendices}

\section{Contents}

A Posterior Conditional Distributions:

Technical details for sampling from the IMIFA model . . . . . . . . . . . . . 1

B Simulation Studies ... . . . . . . . . . . . . . . . . . 4

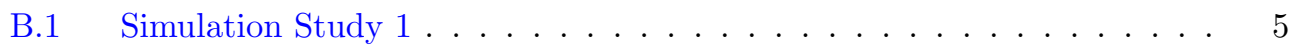

B.2 Simulation Study 2 . . . . . . . . . . . . . . . . . . 7

B.3 Simulation Study 3 . . . . . . . . . . . . . . . . . . 9

C Assessing Robustness of the IMIFA Model . . . . . . . . . . . . . . . . . . . . 11

D Additional Results and Visualisations . . . . . . . . . . . . . . . . 11

\section{A Posterior Conditional Distributions: Technical details for sampling from the IMIFA model}

The structure of the Metropolis-within-Gibbs sampler to conduct inference for the IMIFA model and the exact forms of the required conditional distributions are detailed below. Note that $\mathrm{Ga}(\alpha, \beta)$ refers throughout to the gamma distribution with mean $\alpha / \beta$. The number of observations in a component is denoted by $n_{g}$, where $\mathbf{n}=\left(n_{1}, \ldots, n_{\widetilde{G}}\right)^{\top}$ sums to $N$, and $\widetilde{q}_{g}$ is the current sample of the cluster-specific number of active factors. Algorithms for sampling other models in the IMIFA family can all be considered as special cases of what follows. The algorithm is implemented in the associated R package IMIFA (Murphy et al., 2019). For $g=1, \ldots, \widetilde{G}$, where $\widetilde{G}$ is the current number of active components (of which some may be empty):

$$
\begin{aligned}
& \boldsymbol{\mu}_{g} \mid \ldots \sim \mathrm{N}_{p}\left(\boldsymbol{\Omega}_{\boldsymbol{\mu}}^{-1}\left(\boldsymbol{\Psi}_{g}^{-1}\left(\sum_{i: z_{i g}=1} \mathbf{x}_{i}-\sum_{i: z_{i g}=1} \boldsymbol{\Lambda}_{g} \boldsymbol{\eta}_{i}\right)+\varphi \mathcal{I}_{p} \tilde{\boldsymbol{\mu}}\right), \boldsymbol{\Omega}_{\boldsymbol{\mu}}^{-1}\right), \\
& \boldsymbol{\eta}_{i} \mid z_{i g}=1, \ldots \sim \mathrm{N}_{\widetilde{q}_{g}}\left(\boldsymbol{\Omega}_{\boldsymbol{\eta}}^{-1} \boldsymbol{\Lambda}_{g}^{\top} \boldsymbol{\Psi}_{g}^{-1}\left(\mathbf{x}_{i: z_{i g}=1}-\boldsymbol{\mu}_{g}\right), \boldsymbol{\Omega}_{\boldsymbol{\eta}}\right) \quad \text { for } i=1, \ldots, n_{g}, \\
& \psi_{j g} \mid \ldots \sim \operatorname{IG}\left(\alpha+\frac{n_{g}}{2}, \beta_{j}+\frac{\mathcal{S}_{j g}}{2}\right) \quad \text { for } j=1, \ldots, p, \\
& \boldsymbol{\Lambda}_{j g} \mid \ldots \sim \mathrm{N}_{\widetilde{q}_{g}}\left(\boldsymbol{\Omega}_{\boldsymbol{\lambda}}^{-1} \boldsymbol{\eta}_{i: z_{i g}=1}^{\top} \psi_{j g}^{-1}\left(\mathbf{x}_{i: z_{i g}=1}^{(j)}-\mu_{j g}\right), \boldsymbol{\Omega}_{\boldsymbol{\lambda}}^{-1}\right) \quad \text { for } j=1, \ldots, p,
\end{aligned}
$$




$$
\begin{aligned}
& \phi_{j k g} \mid \ldots \sim \mathrm{Ga}\left(\nu_{1}+\frac{1}{2}, \nu_{2}+\frac{\sigma_{g} \tau_{k g} \lambda_{j k g}^{2}}{2}\right) \quad \text { for } j=1, \ldots, p \text { and } k=1, \ldots, \widetilde{q}_{g}, \\
& \delta_{1 g} \mid \ldots \sim \mathrm{Ga}\left(\alpha_{1}+\frac{p \widetilde{q}_{g}}{2}, \beta_{1}+\frac{\sigma_{g}}{2} \sum_{h=1}^{\widetilde{q}_{g}} \tau_{h g}^{(1)} \sum_{j=1}^{p} \phi_{j h g} \lambda_{j h g}^{2}\right), \\
& \delta_{k g} \mid \ldots \sim \mathrm{Ga}\left(\alpha_{2}+\frac{p}{2}\left(\widetilde{q}_{g}-k+1\right), \beta_{2}+\frac{\sigma_{g}}{2} \sum_{h=k}^{q_{g}} \tau_{h g}^{(k)} \sum_{j=1}^{p} \phi_{j h g} \lambda_{j h g}^{2}\right) \quad \text { for } k=2, \ldots, \widetilde{q}_{g}, \\
& \sigma_{g} \mid \ldots \sim \operatorname{Ga}\left(\varrho_{1}+\frac{p \widetilde{q}_{g}}{2}, \varrho_{2}+\frac{\sum_{k=1}^{\widetilde{q}_{g}} \tau_{k g} \sum_{j=1}^{p} \phi_{j k g} \lambda_{j k g}^{2}}{2}\right), \\
& v_{g} \mid \ldots \sim \operatorname{Beta}\left(1-d+n_{g}, \alpha+g d+N-\sum_{l=1}^{g} n_{l}\right), \\
& u_{i} \mid z_{i g}=1, \ldots \sim \operatorname{Unif}\left(0, \xi_{g}\right) \quad \text { for } i=1, \ldots, N, \\
& \text { where } \quad
\end{aligned}
$$

$$
\begin{aligned}
\boldsymbol{\Omega}_{\boldsymbol{\mu}} & =\varphi \boldsymbol{\mathcal { I }}_{p}+n_{g} \boldsymbol{\Psi}_{g}^{-1} \\
\boldsymbol{\Omega}_{\boldsymbol{\eta}} & =\mathcal{I}_{\widetilde{q}_{g}}+\boldsymbol{\Lambda}_{g}^{\top} \boldsymbol{\Psi}_{g}^{-1} \boldsymbol{\Lambda}_{g}, \\
\boldsymbol{\Omega}_{\boldsymbol{\lambda}} & =\operatorname{diag}\left(\phi_{j 1 g} \tau_{1 g} \sigma_{g}, \ldots, \phi_{j \widetilde{q}_{g} g} \tau_{\widetilde{q}_{g} g} \sigma_{g}\right)+\psi_{j g}^{-1} \boldsymbol{\eta}_{i: z_{i g}=1}^{\top} \boldsymbol{\eta}_{i: z_{i g}=1}, \\
\tau_{h g}^{(k)} & =\prod_{t=1}^{h} \frac{\delta_{t g}}{\delta_{k g}} \\
\pi_{g} & =v_{g} \prod_{l=1}^{g-1}\left(1-v_{l}\right)
\end{aligned}
$$

and

$$
\mathcal{S}_{j g}=\sum_{i: z_{i g}=1}\left(x_{i j}-\mu_{j g}-\boldsymbol{\Lambda}_{j g} \boldsymbol{\eta}_{i}\right)^{\top}\left(x_{i j}-\mu_{j g}-\boldsymbol{\Lambda}_{j g} \boldsymbol{\eta}_{i}\right) .
$$

Here $\mathbf{x}^{(j)}$ denotes the $j$-th column of the data matrix, $\lambda_{j k g}^{2}$ is a single squared loading, and $\tau_{k g}=\prod_{h=1}^{k} \delta_{h g}$ is updated after every update of $\delta_{h g}$.

Parsimonious parameterisations of the component covariance matrices are easily incorporated. Uniquenesses can be constrained to be isotropic, with $\boldsymbol{\Psi}_{g}=\operatorname{diag}\left(\psi_{g}, \ldots, \psi_{g}\right)$, leading to a model that corresponds to an infinite mixture and infinite-dimensional extension of probabilistic principal components analysers (Tipping and Bishop, 1999). Uniquenesses can also be constrained across clusters, with or without the isotropic constraint across variables. These restrictions define the models in the pgmm family (McNicholas and Murphy, 2008) named UUC, UCU, and UCC, respectively, to which the following Gibbs updates are Bayesian analogues 


$$
\begin{aligned}
& \psi_{g} \mid \ldots \sim \operatorname{IG}\left(\alpha+\frac{p n_{g}}{2}, \beta+\frac{\operatorname{tr}\left(\mathcal{S}_{g}\right)}{2}\right), \\
& \psi_{j} \mid \ldots \sim \operatorname{IG}\left(\alpha+\frac{N}{2}, \beta_{j}+\frac{\sum_{g=1}^{G} \mathcal{S}_{j g}}{2}\right), \\
& \psi \mid \ldots \sim \operatorname{IG}\left(\alpha+\frac{p N}{2}, \beta+\frac{\sum_{g=1}^{G} \operatorname{tr}\left(\mathcal{S}_{g}\right)}{2}\right) .
\end{aligned}
$$

In the contexts of finite and overfitted mixtures (i.e. MFA, MIFA, OMFA, and OMIFA) $\mathbf{z}_{i} \mid \mathbf{x}_{i}, \ldots \sim \operatorname{Mult}\left(1, p_{i 1}, \ldots, p_{i \widetilde{G}}\right)$, with

$$
p_{i g}=\operatorname{Pr}\left(z_{i g}=1 \mid \mathbf{x}_{i}, \ldots\right)=\frac{\pi_{g} \mathrm{~N}_{p}\left(\mathbf{x}_{i} ; \boldsymbol{\mu}_{g}, \boldsymbol{\Lambda}_{g} \boldsymbol{\Lambda}_{g}^{\top}+\mathbf{\Psi}_{g}\right)}{\sum_{g=1}^{\widetilde{G}} \pi_{g} \mathrm{~N}_{p}\left(\mathbf{x}_{i} ; \boldsymbol{\mu}_{g}, \boldsymbol{\Lambda}_{g} \boldsymbol{\Lambda}_{g}^{\top}+\mathbf{\Psi}_{g}\right)},
$$

whereas under the IMIFA and IMFA models

$$
p_{i g} \propto \mathrm{N}_{p}\left(\mathbf{x}_{i} ; \boldsymbol{\mu}_{g}, \boldsymbol{\Lambda}_{g} \boldsymbol{\Lambda}_{g}^{\top}+\boldsymbol{\Psi}_{g}\right) \frac{\pi_{g}}{\xi_{g}} \mathbb{1}\left(u_{i}<\xi_{g}\right) .
$$

The allocations $\mathbf{z}_{i}$ are sampled in a fast, numerically stable fashion, using the unnormalised log-probabilities and independent draws from the standard Gumbel distribution (Yellott, 1977) via $s_{i g}=-\ln \left(m_{i g}\right)$, with $m_{i g} \sim \operatorname{Exp}(\lambda=1)$. Observation $i$ is assigned the label $g$ satisfying

$$
\underset{g \in\{1, \ldots, \widetilde{G}\}}{\arg \max }\left\{\ln \left(p_{i g}\right)+s_{i g}\right\} .
$$

For the IMIFA and IMFA models, the sampler need only find the maximum over, and only draw Gumbel noise for, log-probabilities for which the indicator function in (1) evaluates to 1.

Sampling the parameters of the PYP for non-zero $d$ values requires the introduction of Metropolis-Hastings steps within the Gibbs sampler. A joint hyperprior of the form $\mathrm{p}(\alpha, d)=$ $\mathrm{p}(d) \mathrm{p}(\alpha \mid d)$ is assumed, as per Jara et al. (2010). Firstly, the hyperprior for the discount parameter $d$ is similar to the one assumed by Carmona et al. (2019); a mixture of a point-mass at zero and a continuous beta distribution, in order to consider the DP special case with $d=0$ with positive probability, i.e. $d \sim \kappa \delta_{0}+(1-\kappa) \operatorname{Beta}\left(a^{\prime}, b^{\prime}\right)$. This facilitates explicit comparison between DP models and encompassing PYP alternatives. Secondly, the hyperprior for $\alpha$ is given conditionally on $d$, s.t. $(\alpha \mid d) \sim \operatorname{Ga}(\alpha+d ; a, b)$, and includes the constraint $\alpha>-d$ by shifting the support of the gamma density to the interval $(-d, \infty)$; choosing a large $b$ value is particularly relevant as it encourages clustering (Müller and Mitra, 2013).

The likelihood for $\alpha$ and $d$ is given by the exchangeable partition probability function induced by the PYP (Pitman, 1995). Thus, the required conditional posterior distributions are

$$
\begin{aligned}
& \alpha \mid d, \ldots \propto \frac{\Gamma(\alpha+1)}{\Gamma(\alpha+N)}\left\{\prod_{g=1}^{G_{0}-1}(\alpha+g d)\right\} p(\alpha \mid d), \\
& d \mid \alpha, \ldots \propto\left\{\prod_{g=1}^{G_{0}-1}(\alpha+g d)\right\}\left\{\prod_{g=1}^{G_{0}} \frac{\Gamma\left(n_{g}-\alpha\right)}{\Gamma(1-\alpha)}\right\} p(d) .
\end{aligned}
$$


Sampling from the distributions in (2) and (3), while always considering the support $\alpha>-d$, proceeds as per Carmona et al. (2019); a Metropolis-Hastings step is implemented for the discount parameter with independent proposal distribution $0.5 \delta_{0}+0.5 \operatorname{Beta}(d ; 1,1)$, and a random walk Metropolis-Hastings step with proposal distribution given by $\alpha^{\star} \mid \alpha \sim \operatorname{Unif}(\alpha-\zeta, \alpha+\zeta)$ is implemented for the concentration parameter, where $\zeta$ ( $=2$ in our implementation) is used to control the acceptance rate. For $d$, the mutation rate is considered rather than the acceptance rate, whereby a move is only considered accepted if the proposal differs from the current value.

However, when the DP prior is assumed, or when the sampled value of $d$ is exactly zero under the PYP prior, $\alpha$ is updated according to the auxiliary variable routine of West (1992), with Gibbs updates by simulation from a weighted mixture of two gamma distributions, via

$$
\alpha \mid G_{0}, \chi, \ldots \sim \omega_{\chi} \mathrm{Ga}\left(a+G_{0}, b-\ln (\chi)\right)+\left(1-\omega_{\chi}\right) \mathrm{Ga}\left(a+G_{0}-1, b-\ln (\chi)\right),
$$

where $G_{0}$ denotes the current number of non-empty clusters, $\left(\chi \mid \alpha, G_{0}\right) \sim \operatorname{Beta}(\alpha+1, N)$, and the mixing weights $\omega_{\chi}$ are defined by

$$
\frac{\omega_{\chi}}{1-\omega_{\chi}}=\frac{\left(a+G_{0}-1\right)}{N(b-\ln (\chi))}
$$

The complementary label switching moves of Papaspiliopoulos and Roberts (2008), which are effective at swapping similar and unequal clusters, respectively, are also incorporated. Firstly, the labels of two randomly chosen non-empty clusters $g$ and $h$ are swapped with probability $\min \left\{1,\left(\pi_{h} / \pi_{g}\right)^{n_{g}-n_{h}}\right\}$. Secondly, the labels of neighbouring active components $l$ and $l+1$ are swapped with probability $\min \left\{1,\left(1-v_{l+1}\right)^{n_{l}} /\left(1-v_{l}\right)^{n_{l+1}}\right\}$; if accepted, $v_{l}$ and $v_{l+1}$ are also swapped. Cluster-specific parameters are reordered accordingly after each accepted move. Finally, for updating $\alpha$ under the sparse finite OMIFA or OMFA models, with further details in Frühwirth-Schnatter and Malsiner-Walli (2019),

$$
\alpha \mid \mathbf{Z}, \widetilde{G}, \ldots \propto \frac{\Gamma(\alpha \widetilde{G})}{\Gamma(N+\alpha \widetilde{G})}\left\{\prod_{g: n_{g}>0} \frac{\Gamma\left(n_{g}+\alpha\right)}{\Gamma(\alpha)}\right\} p(\alpha) .
$$

\section{B Simulation Studies}

The performance of the novel IMIFA model with its PYP-MGP priors, in terms of inferring both the number of clusters and the cluster-specific numbers of factors, is assessed here through simulation studies. Section B.1 explores sensitivity to the PYP parameters in a range of dimensionality scenarios, with balanced cluster sizes and a common number of factors. The simulation study in Section B.2 is more challenging; a larger number of clusters (many of which are small) are simulated for $N<p$ data, with different numbers of cluster-specific factors (some of which are large). The final simulation study in Section B.3 mirrors the design in Section B.2, only here the true $\boldsymbol{\Lambda}_{g}$ matrices used to generate the data are sparse. 


\section{B.1 Simulation Study 1}

Firstly, data with $G=3$ clusters and $p=50$ variables are simulated with $q_{g}=4 \forall g$, and with $\boldsymbol{\pi}=(1 / 3,1 / 3,1 / 3)$ so that clusters are roughly equally sized. Other model parameters are simulated, with $\boldsymbol{\eta}_{i} \sim \mathrm{N}_{q}\left(\mathbf{0}, \mathcal{I}_{q}\right), \psi_{j g} \sim \mathrm{IG}(2,1)$, and $\boldsymbol{\Lambda}_{j g} \sim \mathrm{N}_{q}\left(\mathbf{0}, \mathcal{I}_{q}\right)$. Notably, loadings are not drawn from the MGP prior (Bhattacharya and Dunson, 2011) underpinning the IMIFA model. To ensure clusters are reasonably closely located, $\boldsymbol{\mu}_{g} \sim \mathrm{N}_{p}\left((2 g-G-1) \mathbf{1}, \boldsymbol{I}_{p}\right)$. The data are then simulated according to the conditional mixture model

$$
f\left(\mathbf{x}_{i} \mid \boldsymbol{\eta}_{i}, \boldsymbol{\theta}\right)=\sum_{g=1}^{G} \pi_{g} \mathrm{~N}_{p}\left(\mathbf{x}_{i} ; \boldsymbol{\mu}_{g}+\boldsymbol{\Lambda}_{g} \boldsymbol{\eta}_{i}, \Psi_{g}\right) .
$$

To evaluate performance in different settings, sample sizes less than, equal to, and greater than $p$ are considered, i.e. $N=25,50$, and 300. Sensitivity to the PYP and DP parameters is explored by firstly assuming a DP prior with various values of $\alpha$ less than, equal to, and greater than 1, and by allowing $\alpha$ to be learned as per West (1992), and secondly by incorporating Metropolis-Hastings steps to learn both $\alpha$ and $d$, assuming a PYP prior.

Results, provided in Table B.1, are based on 10 replicate data sets, standardised prior to model fitting, for each scenario. MCMC chains were run for 25,000 iterations, with every $2^{\text {nd }}$ sample thinned and the first $20 \%$ of iterations discarded as burn-in. Cluster labels were initialised using mclust (Scrucca et al., 2016), as hierarchical clustering gave poor, heavily imbalanced starting values. As the cluster-specific $\boldsymbol{\Lambda}_{g}$ and $\boldsymbol{\Psi}_{g}$ parameters could still induce separation among clusters, pairwise scatterplots from one randomly chosen raw replicate data set under the $N>p$ scenario are shown in Figure B.1 to demonstrate the extent of the overlap; for visual clarity, only 5 randomly chosen variables are depicted.

Table B.1: Aggregated simulation study results for the IMIFA model under different dimensionality scenarios and settings of the concentration and discount parameters $\alpha$ and $d$ (posterior mean estimates thereof in parentheses where appropriate). The modal estimates of $G$ and associated estimates of $q_{g} \forall g$ are reported (with $95 \%$ credible intervals in brackets). Clustering performance is assessed through the average percentage error rate against the known cluster labels.

\begin{tabular}{c|c|c|c|c|c|c|c} 
Dimension & $\alpha$ & $d$ & $G$ & $q_{1}$ & $q_{2}$ & $q_{3}$ & Error $(\%)$ \\
\hline & 0.5 & 0 & $3[3,3]$ & $5[3,9]$ & $5[3,9]$ & $5[3,9]$ & 0 \\
$N=25$ & 1 & 0 & $3[3,3]$ & $5[3,9]$ & $5[3,9]$ & $5[3,9]$ & 0 \\
$(N<p)$ & 5 & 0 & $3[3,4]$ & $5[3,9]$ & $5[3,9]$ & $5[3,9]$ & 6.4 \\
& $(0.57)$ & 0 & $3[3,3]$ & $5[3,9]$ & $5[3,9]$ & $5[3,9]$ & 0 \\
& $(0.51)$ & $(0.05)$ & $3[3,3]$ & $5[3,9]$ & $5[3,9]$ & $5[3,9]$ & 0 \\
\hline & 0.5 & 0 & $3[3,3]$ & $5[4,7]$ & $5[4,7]$ & $5[4,7]$ & 0 \\
$N=50$ & 1 & 0 & $3[3,3]$ & $5[4,7]$ & $5[4,7]$ & $5[4,7]$ & 0 \\
$(N=p)$ & 5 & 0 & $3[3,3]$ & $5[4,7]$ & $5[4,7]$ & $5[4,7]$ & 0 \\
& $(0.52)$ & 0 & $3[3,3]$ & $5[4,7]$ & $5[4,7]$ & $5[4,7]$ & 0 \\
& $(0.48)$ & $(0.03)$ & $3[3,3]$ & $5[4,7]$ & $5[4,7]$ & $5[4,7]$ & 0 \\
\hline \multirow{2}{*}{$N=300$} & 0.5 & 0 & $3[3,3]$ & $5[4,6]$ & $5[4,6]$ & $5[4,6]$ & 0 \\
$(N>p)$ & 1 & 0 & $3[3,3]$ & $5[4,6]$ & $5[4,6]$ & $5[4,6]$ & 0 \\
& 5 & 0 & $3[3,3]$ & $5[4,6]$ & $5[4,6]$ & $5[4,6]$ & 0 \\
& $(0.42)$ & 0 & $3[3,3]$ & $5[4,6]$ & $5[4,6]$ & $5[4,6]$ & 0
\end{tabular}



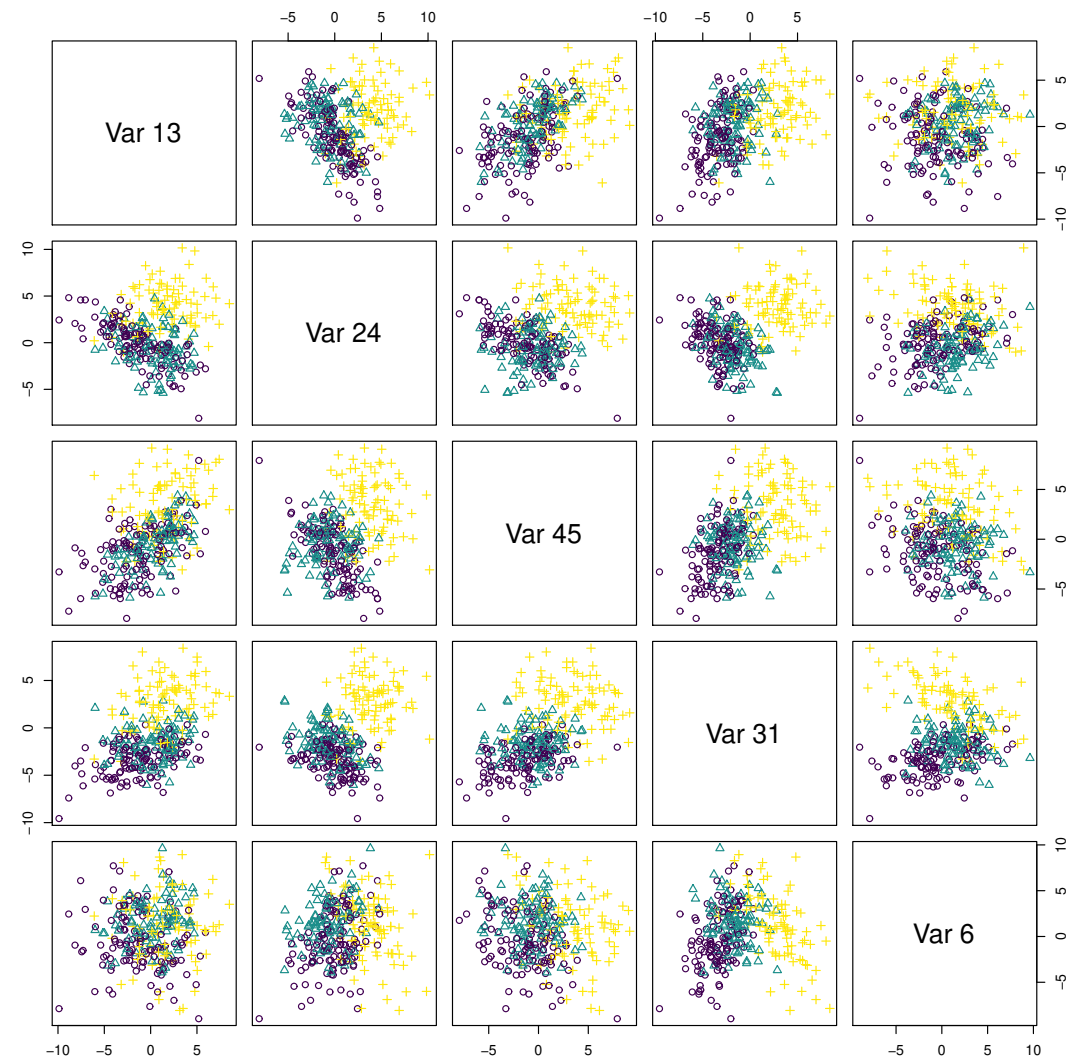

Figure B.1: Pairwise scatterplots of 5 randomly chosen variables from one of the raw replicate data sets under the $N>p$ scenario in Table B.1, demonstrating the overlap between the 3 clusters.

Table B.1 clearly demonstrates that the IMIFA model performs well overall for these data, exhibiting capability to uncover the structure within the simulated data sets regardless of dimensionality. The modal estimate of $G$ is equal to the truth in all cases, with only the $N<p$, $\alpha=5$ scenario showing some deviation in the $95 \%$ credible interval. Clustering performance is mostly perfect. Furthermore, in every case, the true value of $q_{g}=4$ is within the limits of the associated credible intervals, which intuitively become narrower as more data accumulates. While the modal estimates $\widehat{q}_{g}$ are consistently greater than the truth throughout Table B.1, overestimation should be preferred to underestimation; a less parsimonious model which nevertheless fits well and uncovers the true clustering structure is better than one which loses information and fits poorly due to having too few factors. Recall that the loadings were drawn from a standard multivariate Gaussian, rather than the MGP prior underpinning the IMIFA model, i.e. entries in the true $\boldsymbol{\Lambda}_{g}$ matrices did not shrink with the column index, nor were the loadings sparse. Thus, there is evidence to suggest the model is liable to overestimate the number of factors when the $\boldsymbol{\Lambda}_{g}$ matrices, and by extension the cluser-specific marginal covariance matrices, are dense. This is explored further in the subsequent simulation studies. 


\section{B.2 Simulation Study 2}

Results of a more challenging simulation study are presented in Figure B.3; here, $N<p$ data $(N=200, p=250)$ are simulated with a large number of clusters and uncommon numbers of cluster-specific factors. In particular, many of the $G=10$ clusters are small (a setting often studied in Bayesian nonparametric modelling), with $\boldsymbol{\pi}=(0.25,0.2,0.15,0.1,0.05, \ldots, 0.05)^{\top}$. The numbers of factors $q_{1}, \ldots, q_{g}$ are drawn randomly from $0, \ldots, \min \left(15, n_{g}-1\right)$, where the upper limit ensures that no cluster has more factors than observations. Otherwise, the same parameter settings as Simulation Study 1 above (Section B.1) were used to generate the data.

Results of fitting an IMIFA model assuming a PYP prior, allowing both $\alpha$ and $d$ to be learned, and otherwise using the same sampler settings as in Section B.1 above, are given for 5 replicates of this scenario, with the $\boldsymbol{\pi}$ vector ordered randomly for each data set. To demonstrate the extent of the challenge these settings represent, pairwise scatterplots are again shown for 5 randomly chosen variables for the first replicate data set in Figure B.2.

Figure B.3 shows that the model over-estimates the number of clusters, though in some cases the ARI values are nonetheless quite good, as the larger clusters are generally uncovered well. However, the smaller clusters are further divided, albeit cleanly, into smaller sub-clusters with, in some cases, just 1 or 2 units inside. In these cases, the modal $\widehat{q}_{g}$ estimates are close or equal to the upper limit of the adaptive Gibbs sampler $(3 \ln (p))$, and hence or otherwise greater than the corresponding estimated cluster sizes $\widehat{n}_{g}$. Thus, there is evidence that the model has difficulty in adaptively shrinking the $\boldsymbol{\Lambda}_{g}$ matrices when there are many clusters with few units.

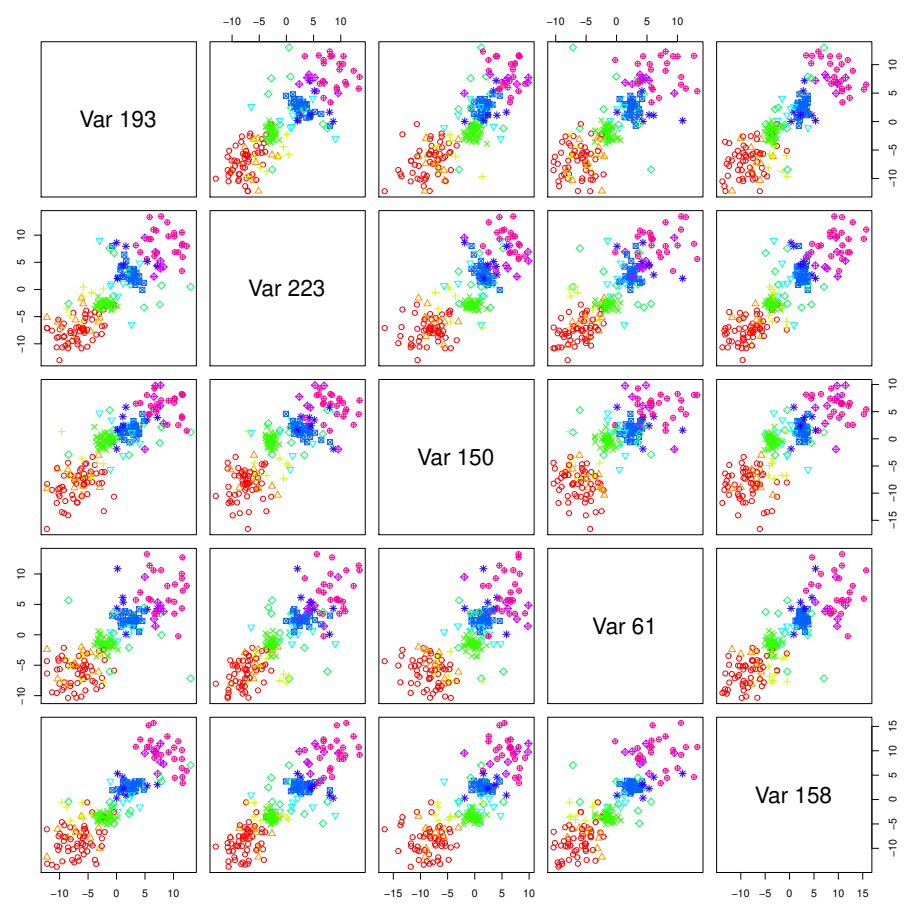

Figure B.2: Pairwise scatterplots of 5 randomly chosen variables from the first raw replicate data set in Simulation Study 2 (Section B.2), demonstrating the overlap between the 10 clusters. 

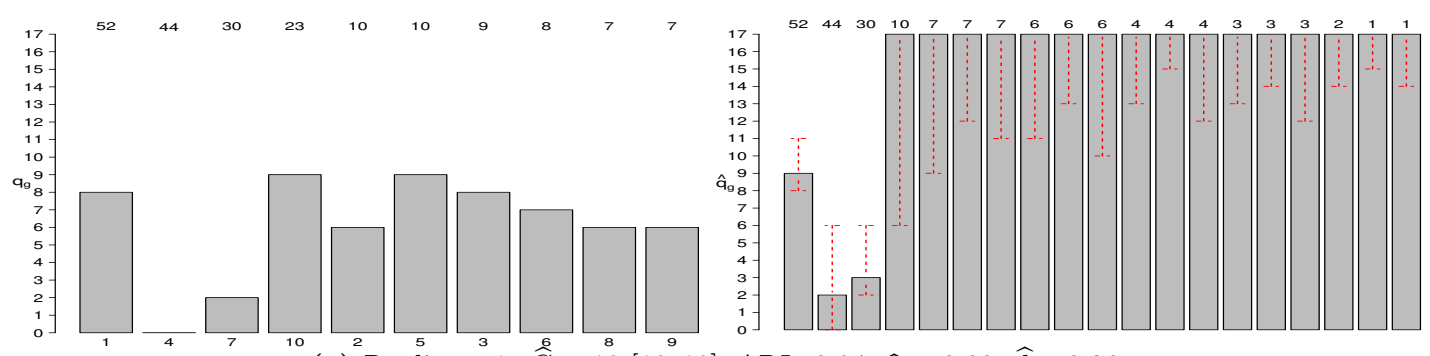

(a) Replicate 1: $\widehat{G}=19$ [19, 19], ARI $=0.94, \widehat{\alpha}=0.80, \widehat{d}=0.26$.
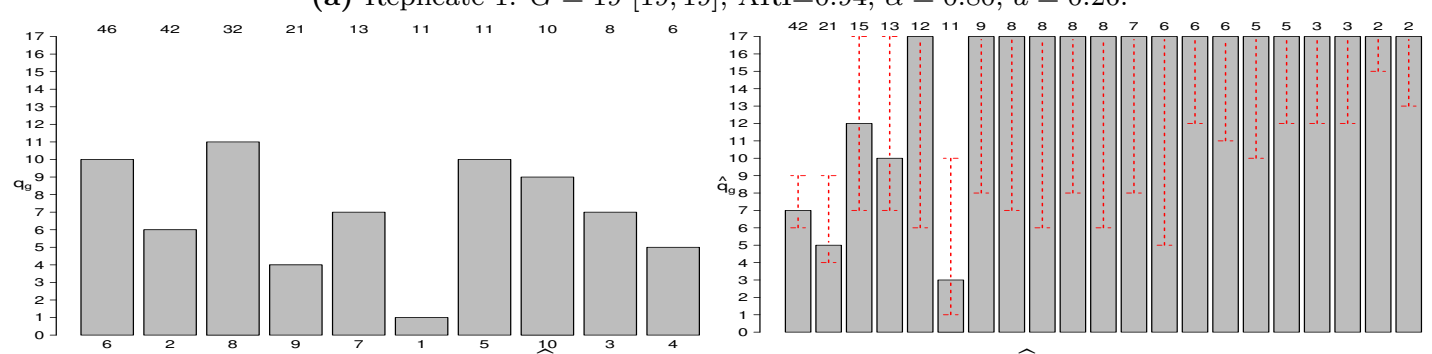

(b) Replicate 2: $\stackrel{10}{G}=21[21,22], \mathrm{ARI}=0.66, \widehat{\alpha}=1.20, \widehat{d}=0.20$.
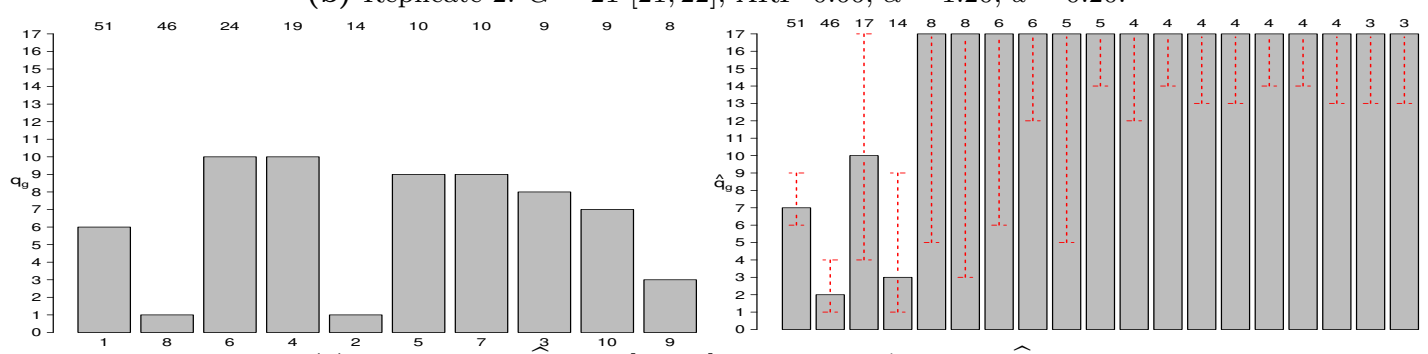

(c) ${ }^{2}$ Replicate 3: $\widehat{G}^{3}=19^{10}[19,24]$, ARI $=0.90, \widehat{\alpha}=1.06, \widehat{d}=0.21$.
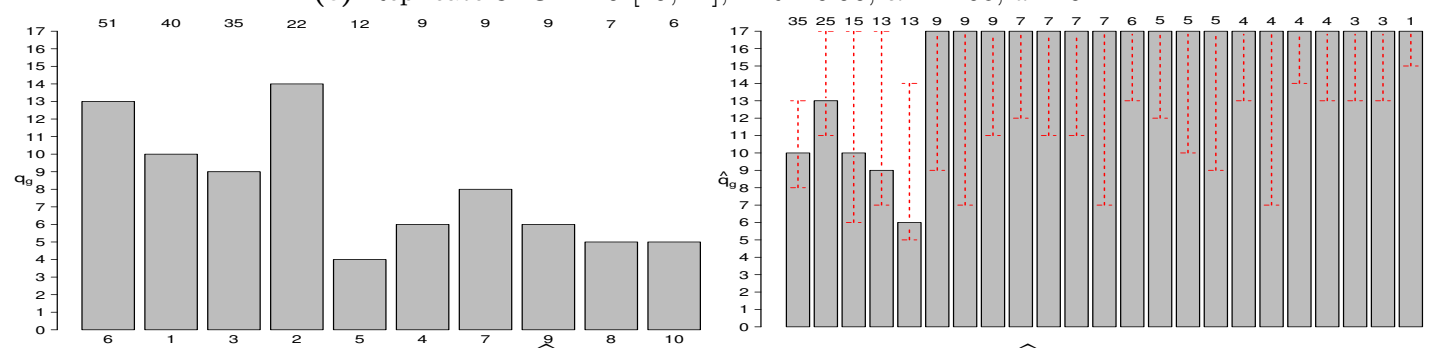

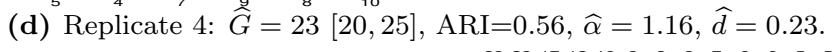
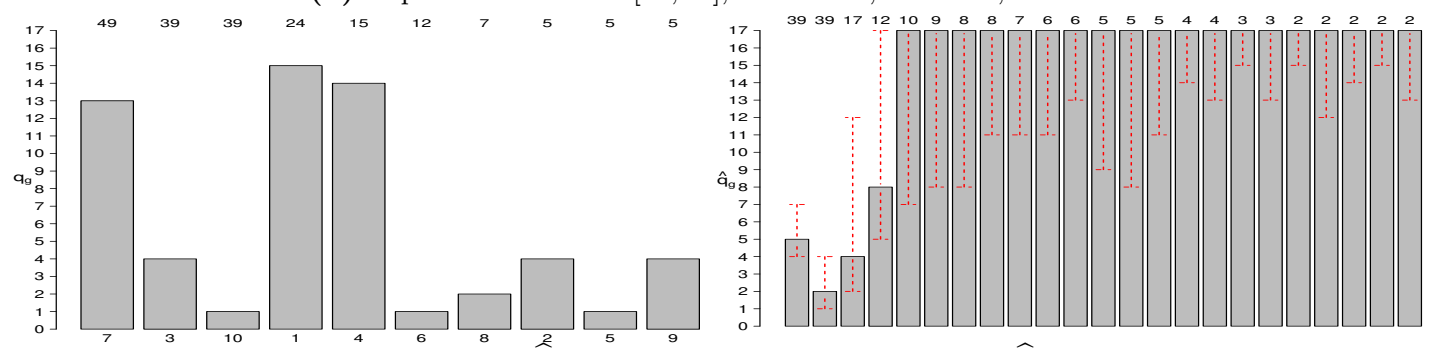

(e) Replicate 5: $\stackrel{4}{G}^{\frac{4}{G}}=23$ [23, 23], ARI $=0.68, \widehat{\alpha}=0.92, \widehat{d}=0.26$.

Figure B.3: Barplots of the true number of cluster-specific factors $q_{g}$ (left) and estimates $\widehat{q}_{g}$ (right) for each replicate data set and corresponding fitted IMIFA model comprising Simulation Study 2. Bars are sorted in descending order of $n_{g}$ and $\widehat{n}_{g}$, respectively, and labelled above with these true and estimated cluster sizes. The plots on the left are also labelled below with the cluster indices. Vertical red lines in the plots on the right show $95 \%$ credible intervals for $\widehat{q}_{g}$. Modal $\widehat{G}$ estimates (with $95 \%$ credible intervals in brackets), ARI values, and posterior mean estimates $\widehat{\alpha}$ and $\widehat{d}$ are given for each replicate. 


\section{B.3 Simulation Study 3}

In both previous simulation studies, the true loadings were dense, having been drawn from a standard multivariate Gaussian, rather than the MGP prior underpinning the model. The design of this final simulation study exactly mirrors the parameter and sampler settings used in Section B.2 with the sole exception that, as per the simulation study design in Bhattacharya and Dunson (2011), the true loadings matrices used to generate the data are sparse. Specifically, the number of non-zero loadings in each $\boldsymbol{\Lambda}_{g}$ matrix begins at $p$ in column 1 , and successively decays by $10 \%$ for each subsequent column. The locations of the zeros in each column are allocated randomly and non-zero elements are drawn from a standard multivariate Gaussian. Again, pairwise scatterplots are shown for 5 randomly chosen variables for the first of the five replicate data sets in Figure B.4, to demonstrate the extent of the overlap between clusters.

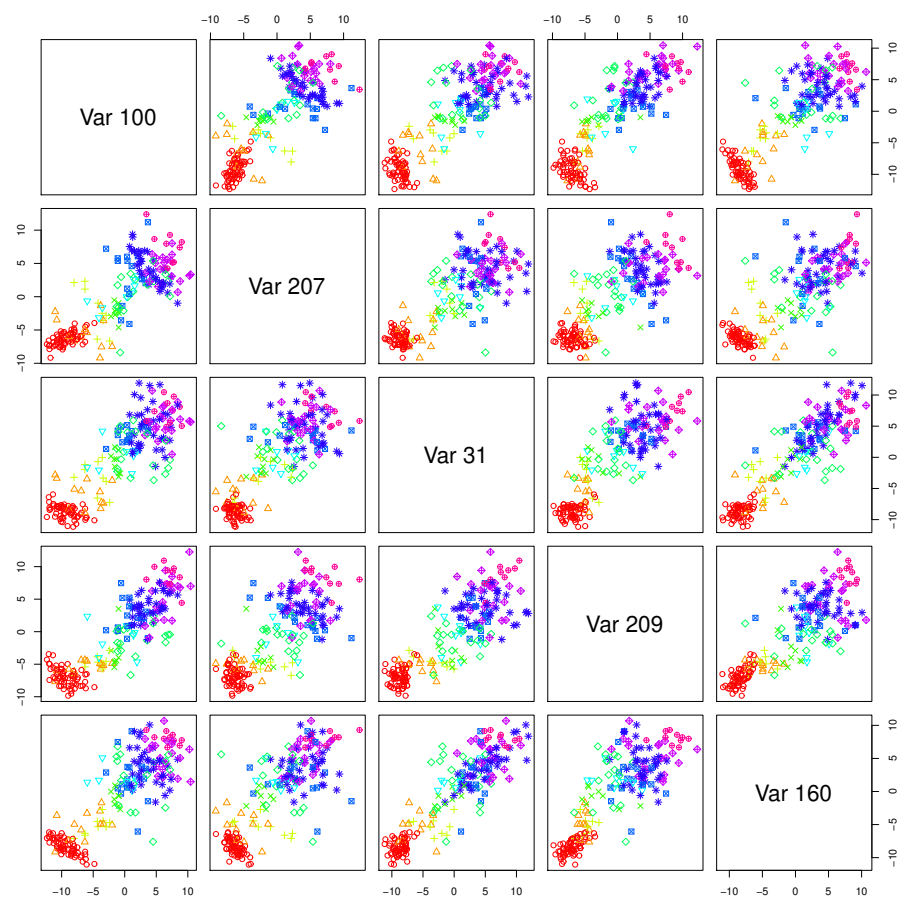

Figure B.4: Pairwise scatterplots of 5 randomly chosen variables from the first raw replicate data set in Simulation Study 3 (Section B.3), demonstrating the overlap between the 10 clusters.

Results are presented in Figure B.5. Performance is comparable to the results of Simulation Study 2, in the sense that, again, the number of clusters is over-estimated. ARI values are nonetheless acceptable. Small clusters are divided into even smaller sub-clusters for which the model struggles to adaptively shrink the number of factors. The comparability of the results of these experiments suggests that performance is being driven not by whether the loadings used to generate the data exhibit increasing levels of sparsity across columns, in line with the MGP prior underpinning the model, but by the presence of many small clusters. The over-estimation of $\widehat{q}_{g}$ in the small clusters in simulation studies 2 and 3 suggests that the hyperparameters $\alpha_{1}$ and $\alpha_{2}$ related to the MGP column shrinkage parameters may need to be higher in mixture settings to enforce a greater degree of shrinkage as there will be fewer data in each cluster from which local and global shrinkage parameters can be learned, compared to fitting an IFA model on the full data set. Introducing Metropolis-Hastings steps to allow these hyperparameters be cluster-specific and learned from the data, rather than fixed, may also help in this regard. 

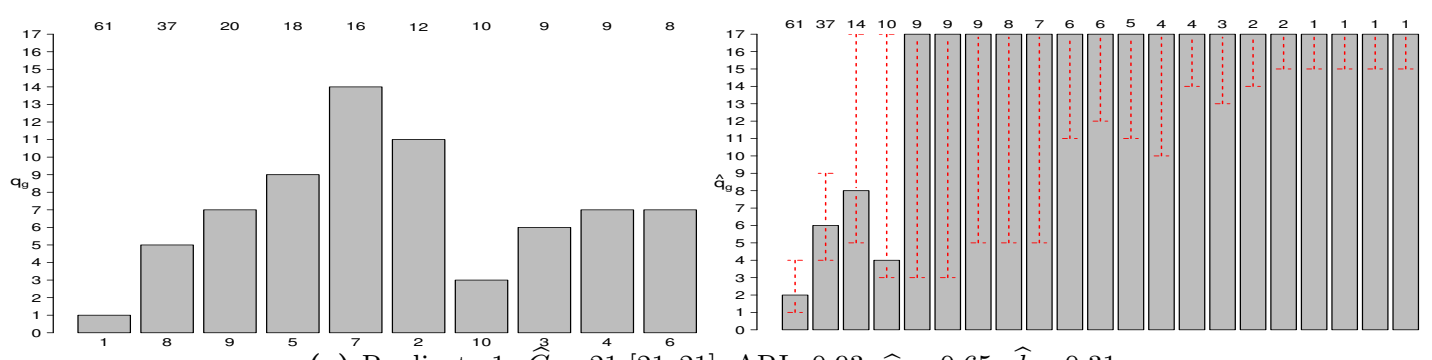

(a) Replicate 1: $\widehat{G}=21[21,21], \mathrm{ARI}=0.93, \widehat{\alpha}=0.65, \widehat{d}=0.31$.
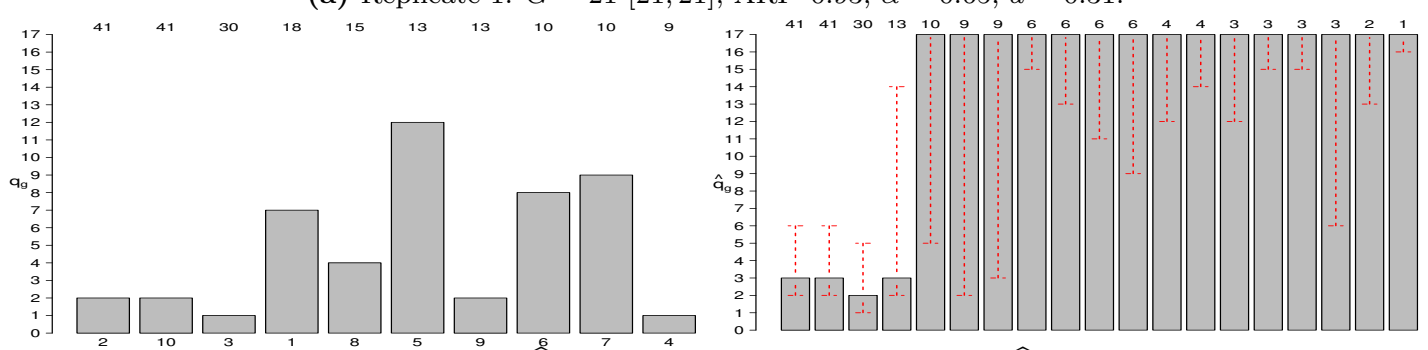

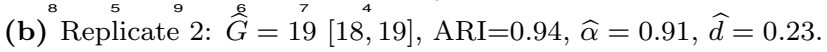
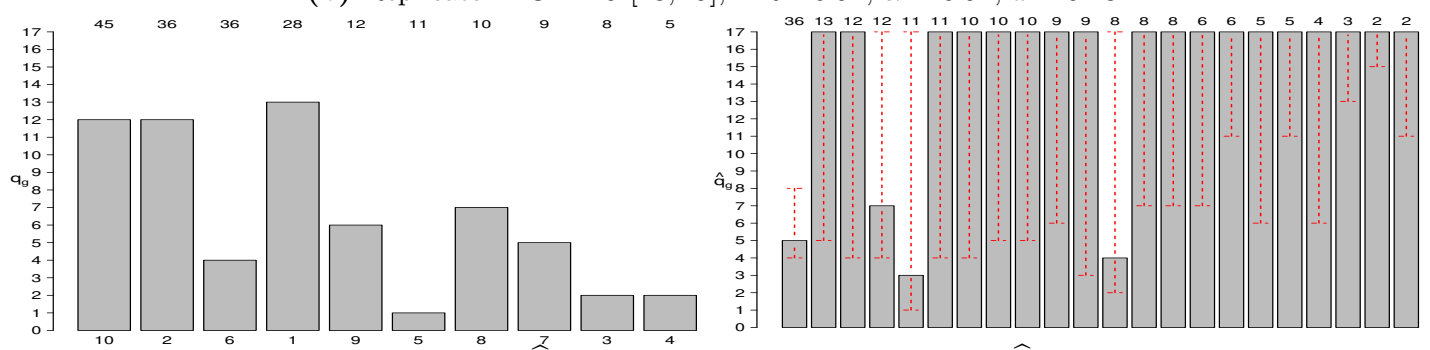

(c) Replicate 3: $\widehat{G}^{\frac{3}{3}}=22$ [22, 22], ARI $=0.59, \widehat{\alpha}=1.35, \widehat{d}=0.18$.
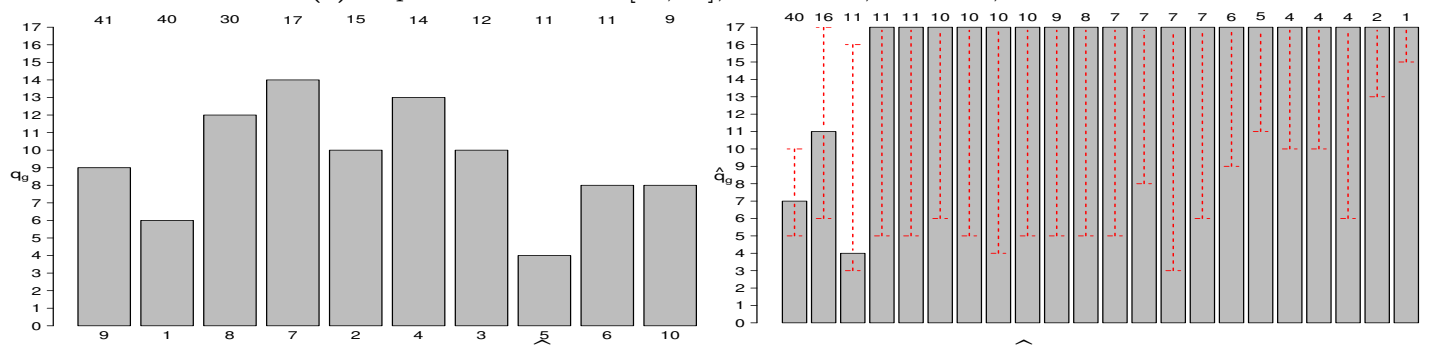

(d) ${ }^{2}$ Replicate 4: $\widehat{G}=22[22,22]$, ARI $=0.69, \widehat{\alpha}=1.22, \widehat{d}=0.21$.
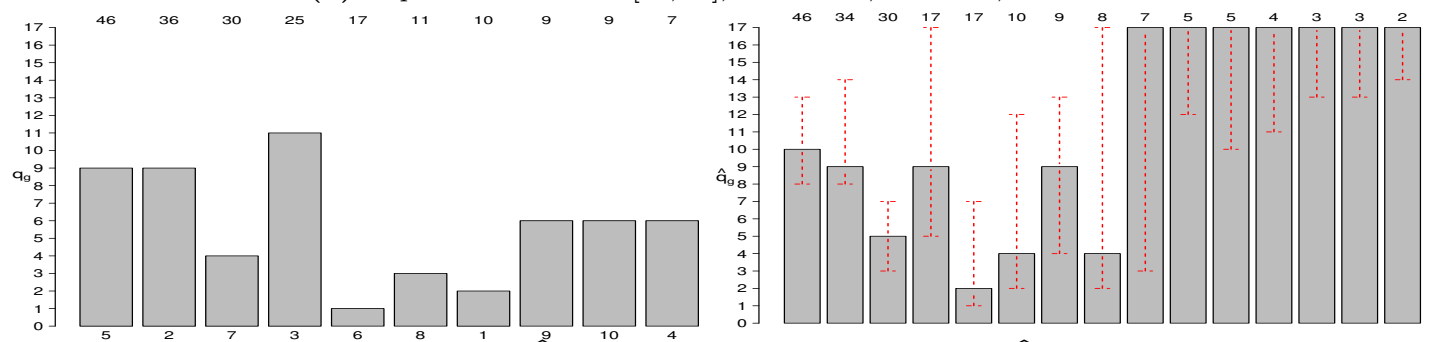

(e) ${ }^{6}$ Replicate 5: $\stackrel{8}{G}=15^{10}[14,25]$, ARI $=0.94, \widehat{\alpha}=1.09, \widehat{d}=0.18$.

Figure B.5: Barplots of the true number of cluster-specific factors $q_{g}$ (left) and estimates $\widehat{q}_{g}$ (right) for each replicate data set and corresponding fitted IMIFA model comprising Simulation Study 3. Bars are sorted in descending order of $n_{g}$ and $\widehat{n}_{g}$, respectively, and labelled above with these true and estimated cluster sizes. The plots on the left are also labelled below with the cluster indices. Vertical red lines in the plots on the right show $95 \%$ credible intervals for $\widehat{q}_{g}$. Modal $\widehat{G}$ estimates (with $95 \%$ credible intervals in brackets), ARI values, and posterior mean estimates $\widehat{\alpha}$ and $\widehat{d}$ are given for each replicate. 


\section{Assessing Robustness of the IMIFA Model}

In order to assess the robustness of the IMIFA model, $N(0,1)$ noise with no clustering information was appended separately to the rows and columns of the olive oil data set. Six new scenarios were generated with 10, 50, and 100 extra variables, and the same numbers of extra observations. Cluster validity is evaluated in Table C. 1 with respect to the 4 area relabelling in Table 3b. In the case of extra observations, noise observations are labelled as though they belong to a fifth cluster. Data were mean-centred and unit-scaled only after expansion.

As the number of irrelevant variables increases, the clustering structure can still be uncovered quite well, however mixing becomes slower and there is increasing support for clusters with only one or no factors as the signal-to-noise ratio decreases. As such, variable selection, or at least data pre-processing, may still be required. As rows of noise are appended, IMIFA generally has no difficulty in assigning these observations to a cluster of their own. Interestingly, clusters corresponding to noise observations correctly require no latent factor structure.

Table C.1: Clustering performance of the IMIFA model on expanded noisy versions of the Italian olive oil data. The run-time relative to running IMIFA on the original data, posterior mean of the PYP parameters $\alpha$ and $d$, modal estimates of $G$ and $\mathbf{Q}$, ARI, and percentage error rate are all given.

\begin{tabular}{c|c|c|c|c|c|c|c} 
Scenario & Relative Time & $\alpha$ & $d$ & $G$ & $\mathbf{Q}$ & ARI & Error $(\%)$ \\
\hline$N=572, p=18$ & 1.86 & 0.48 & 0.01 & 4 & $3,4,4,3$ & 0.85 & 12.59 \\
$N=572, p=58$ & 3.14 & 0.47 & 0.01 & 4 & $1,2,2,2$ & 0.74 & 14.69 \\
$N=572, p=108$ & 5.64 & 0.46 & 0.02 & 4 & $0,1,0,2$ & 0.73 & 17.66 \\
\hline$N=582, p=8$ & 1.10 & 0.57 & 0.01 & 5 & $6,2,2,2,0$ & 0.94 & 6.87 \\
$N=622, p=8$ & 1.09 & 0.56 & 0.01 & 5 & $4,1,1,2,0$ & 0.95 & 6.59 \\
$N=672, p=8$ & 1.07 & 0.53 & 0.01 & 5 & $4,1,2,2,0$ & 1.00 & 0.45
\end{tabular}

\section{Additional Results and Visualisations}

In this Section, some additional visualisations of the results of the illustrative applications are provided. Specifically, more details are provided on the posterior predictive model fit assessment and the observation-specific cluster membership uncertainties. All plots were produced using the associated R package IMIFA (Murphy et al., 2019).

The Posterior Predictive Reconstruction Error (PPRE) has been proposed as a posterior predictive checking strategy for models in the IMIFA family. In short, this involves computing the standardised Frobenius norm of the difference between a matrix of histogram bin counts for the modelled data set and similar matrices constructed using replicate data drawn from the posterior predictive distribution. While the median PPRE value or boxplots of the distribution of PPRE values have been shown to yield useful global measures of model fit in multivariate settings, the histograms themselves can also be studied on a variable-by-variable basis. Due to the high dimensionality of the spectral metabolomic and USPS digits data sets, Figure D.1 shows only the histograms comparing bin counts for the $p=8$ variables in the standardised Italian olive oil data, to which an IMIFA model was fitted, against corresponding counts for the replicate data under the fitted IMIFA model. The true bin counts are within the $95 \%$ credible intervals of the replicate data bin counts in the vast majority of cases, indicating good model fit: recall that this IMIFA model achieves a median PPRE of just 0.10.

The IMIFA model fitted to the USPS digits data set uncovers $\widehat{G}=21$ clusters. Regarding the uncertainty in the allocations to these clusters, the model-based nature of IMIFA facilitates estimation of the uncertainty with which observation $i$ is assigned to its cluster $g$ via 


$$
\widehat{U}_{i}=\min _{g \in\{1, \ldots, \widehat{G}\}}\left\{1-\widehat{z}_{i g}\right\},
$$

where $\widehat{z}_{i g}$ is the estimated probability that observation $i$ belongs to cluster $g$. Figure D.2 shows that the observation-specific cluster membership uncertainties are generally quite low, with the mean uncertainty being just 0.02 and $92 \%$ of observations being assigned with uncertainty less than $1 / \widehat{G}$. A similar plot for the olive oil data is shown in the main text (Figure 4); uncertainties for the spectral metabolomic data are not shown, as there was no uncertainty in the assignments under the fitted IMIFA model (i.e. $\widehat{U}_{i}=0 \forall i=1, \ldots, N$ ).
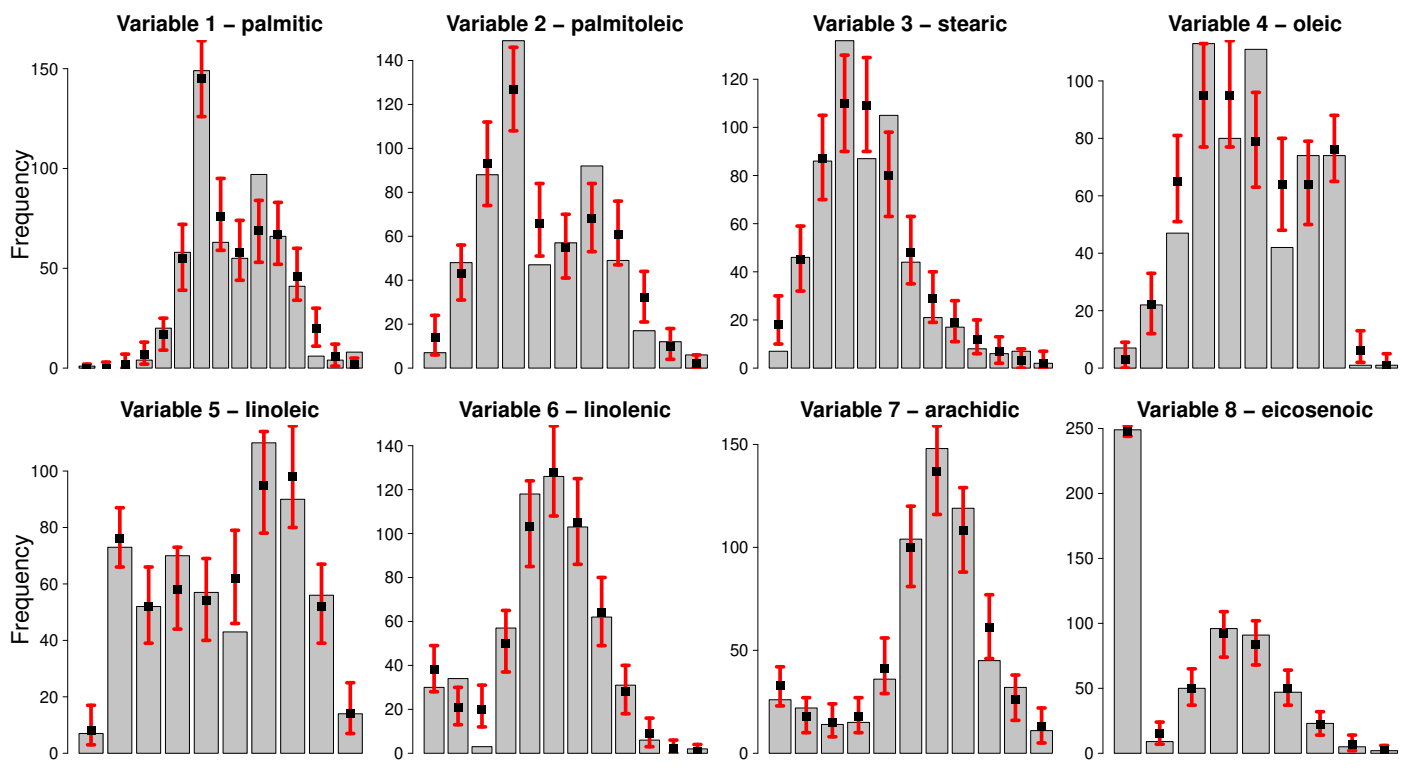

Figure D.1: Histograms of the $p=8$ variables in the standardised Italian olive oil data set. The height of each bar corresponds to the modelled data set, while the black squares correspond to the median bin counts of the replicate data sets drawn from the posterior predictive distribution of the fitted IMIFA model (with associated $95 \%$ credible intervals given by vertical red lines).

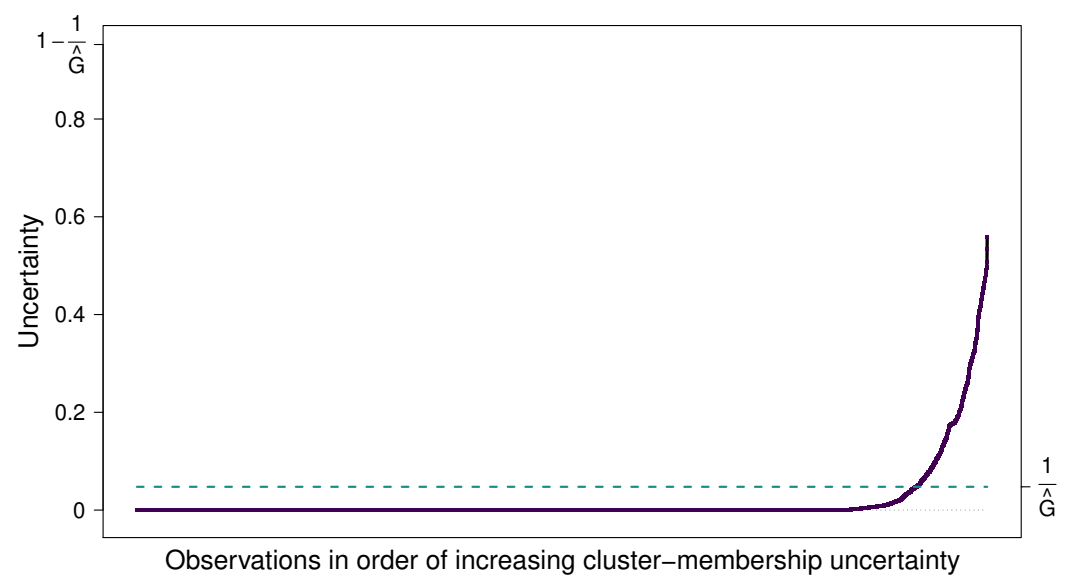

Figure D.2: Uncertainty profile plot for the 21-cluster IMIFA model fitted to the USPS digits data, showing observation-specific uncertainties in increasing order, most of which are below the line at $1 / \widehat{G}$. 


\section{References}

Bhattacharya, A. and Dunson, D. B. (2011). "Sparse Bayesian infinite factor models." Biometrika, 98(2): 291-306. 5, 9

Carmona, C., Nieto-barajas, L., and Canale, A. (2019). "Model based approach for household clustering with mixed scale variables." Advances in Data Analysis and Classification, 13(2): 559-583. 3, 4

Frühwirth-Schnatter, S. and Malsiner-Walli, G. (2019). "From here to infinity: sparse finite versus Dirichlet process mixtures in model-based clustering." Advances in Data Analysis and Classification, 13(1): 33-63. 4

Jara, M., Lesaffre, E., De Iorio, M., and Quintana, F. (2010). "Bayesian semiparametric inference for multivariate doubly-interval-censored data." The Annals of Applied Statistics, 4(4): $2126-2149.3$

McNicholas, P. D. and Murphy, T. B. (2008). "Parsimonious Gaussian mixture models." Statistics and Computing, 18(3): 285-296. 2

Müller, P. and Mitra, R. (2013). "Bayesian nonparametric inference - why and how." Bayesian Analysis, 8(2): 269-360. 3

Murphy, K., Viroli, C., and Gormley, I. C. (2019). IMIFA: infinite mixtures of infinite factor analysers and related models. $\mathrm{R}$ package version 2.1.0.

URL https://cran.r-project.org/package $=$ IMIFA 1, 11

Papaspiliopoulos, O. and Roberts, G. O. (2008). "Retrospective Markov chain Monte Carlo methods for Dirichlet process hierarchical models." Biometrika, 95(1): 169-186. 4

Pitman, J. (1995). "Exchangeable and partially exchangeable random partitions." Probability Theory and Related Fields, 102(2): 145-158. 3

Scrucca, L., Fop, M., Murphy, T. B., and Raftery, A. E. (2016). "mclust 5: clustering, classification and density estimation using Gaussian finite mixture models." The $R$ Journal, $8(1): 289-317.5$

Tipping, M. E. and Bishop, C. M. (1999). "Mixtures of probabilistic principal component analyzers." Neural Computation, 11(2): 443-482. 2

West, M. (1992). "Hyperparameter estimation in Dirichlet process mixture models." Technical report, ISDS Discussion Paper 92-A03, Duke University. 4, 5

Yellott, J. I., Jr. (1977). "The relationship between Luce's choice axiom, Thurstone's theory of comparative judgment, and the double exponential distribution." Journal of Mathematical Psychology, 15(2): 109-144. 3 